\title{
AP-2 Family of Transcription Factors: Critical Regulators of Human Development and Cancer
}

\author{
Yi-Liu Yang' ${ }^{\prime}$ Lin-Yong Zhao ${ }^{2 *}$ \\ 'West China School of Medicine, West China Hospital, Sichuan University, Chengdu, China \\ ${ }^{2}$ Department of Gastrointestinal Surgery and Laboratory of Gastric Cancer, State Key Laboratory of Biotherapy, West China Hospital, Sichuan University, and \\ Collaborative Innovation Center for Biotherapy, Chengdu, China
}

Article Info

\section{Article Notes}

Received: November 24, 2020

Accepted: January 15, 202

\section{*Correspondence:}

Dr. Lin-Yong Zhao, Department of Gastrointestinal Surgery and Laboratory of Gastric Cancer, State Key Laboratory of Biotherapy, West China Hospital, Sichuan University, and Collaborative Innovation Center for Biotherapy, №. 37 Guo Xue Xiang Street, Chengdu, Sichuan Province, China; Email: 153795352@scu.edu.cn.

(c) 2021 Zhao LY. This article is distributed under the terms of the Creative Commons Attribution 4.0 International License.

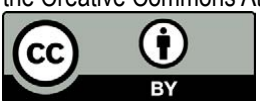

\section{Keywords:}

Transcription factors

AP-2 family

Embryogenesis

Cancer

Tumorigenesis

Prognosis

\begin{abstract}
The AP-2 family of transcription factors consist of DNA-binding proteins: AP- $2 \alpha$ to AP- $2 \varepsilon$. Members and homologs of this family are also known in frogs, fish and invertebrates. These proteins have the same central basic region and a helix-span-helix dimerization motif, which is necessary for dimerization and DNA binding. This family have been found to influence facial, limbs and kidney development in embryogenesis while regulating differentiation and apoptosis. These proteins are also involved in regulation of endocrine processes. In addition to their influence on growth and development, this family have also been reported to correlate with tumorigenesis and development of cancer. At present, this family have been related to tumors of ovary, melanoma, lung, nasopharynx, breast, glioma, neuroblastoma, colon, etc. They regulate expression of many cancer-related genes and affect the occurrence, development, invasiveness and therapeutic response of cancers. Different expression levels of AP-2s are also related to different survival rate. These findings may bring new idea to the diagnosis, classification, treatment and prognosis of cancer.
\end{abstract}

The AP-2 transcription factors family (AP-2 family) constitute five DNA-binding proteins: AP- $2 \alpha$ to AP- $2 \varepsilon$, which are encoded by TFAP2A to TFAP2E, respectively. Frogs, fish and invertebrates also have some members or homologs of this family. Except AP-2 $\delta$, the other four of the family are encoded by seven exons and have the same central basic region and a helix-span-helix dimerization motif, which is necessary for dimerization and DNA binding ${ }^{1}$. In addition to its highly similar DNA-binding and dimerization domains with other family members, AP- $2 \delta$ has unique sequence specificity which has not been observed in other four proteins. This may be useful for regulation of target gene activation ${ }^{2}$.

AP-2 family play important roles in regulating cell differentiation and apoptosis. They have been found to influence body development in embryogenesis, including formation of face, limbs, kidney, retina, central nervous system, and heart ${ }^{3,4}$. Mutations or defects of TFAP2A and TFAP2B lead to developmental malformation ${ }^{5,6}$. These genes are also involved in regulation of endocrine processes. For instance, $T F A P 2 B$ is associated with insulin resistance and diabetes ${ }^{7}$, while TFAP2C plays a key role in regulating genes of estrogen signaling 8 .

In addition to their effect on cell-fate deciding and development, AP-2 family have also been reported to be involved in tumorigenesis and development of cancer. By far, this family have been found to be closely associated with various tumors, including ovarian cancer, 
melanoma, lung cancer, nasopharygeal cancer, breast cancer, glioma, neuroblastoma, gastric cancer, colon cancer, ect. $^{9-17}$. They regulate the expression of many cancerrelated genes, especially in breast cancer. Moreover, they coordinate with the occurrence, development, invasiveness and therapeutic response of cancers. Different expression levels of AP-2s are also related to different survival rate.

\section{AP-2 $\alpha$}

AP- $2 \alpha$ encoded by TFAP $2 A$ regulates cell growth and tissue differentiation. Its expression has been observed in epithelial and neural crest cell lineages in early stage of murine embryogenesis ${ }^{3}$. Mutations of TFAP2A have been found to result in Branchio-oculo-facial syndrome (BOFS), a rare orofacial cleft syndrome which includes cutaneous, ocular, renal and ectodermal anomalies, along with characteristic facial appearance ${ }^{5}$.

Different expression levels of AP- $2 \alpha$ have also been reported in cancer cells.

Overexpression of AP- $2 \alpha$ has been found in tumors of ovary, nasopharynx and lung, and the increased expression may promote tumorigenesis and lead to deteriorate outcome for cancers. In epithelial cells of normal ovary, $\mathrm{AP}-2 \alpha$ protein is only expressed in the cytoplasm. But in malignant epithelial ovarian tumors, AP- $2 \alpha$ is expressed both in the nucleus and cytoplasm. The expression level of AP- $2 \alpha$ in the nucleus is related to increased risk of dying ${ }^{9}$. High expression of AP- $2 \alpha$ has also been reported in nasopharyngeal carcinoma cells, which promotes tumor growth, whereas the downregulation of AP- $2 \alpha$ expression inhibits cell viability and suppresses tumor growth along with microvessel density ${ }^{14}$. The up-regulated expression level is also found in lung carcinoma, which is highly associated with poor prognosis ${ }^{12}$. This outcome is consistent with another study, in which TFAP2A upregulates the expression of KRT16, an independent prognosis predictor related to bad survival for lung cancer ${ }^{18}$.

In some other cancers, however, the expression of AP$2 \alpha$ is decreased, which is related to tumor progression. For example, suppressed expression of AP- $2 \alpha$ in breast cancer seems to occur more frequently in invasive breast tumors than in ductal carcinoma in situ ${ }^{19}$. Similarly, AP- $2 \alpha$ expression correlates inversely with glioma grade, which may suggest its direct role in glioma tumorigenicity ${ }^{11}$ The association between reduced AP- $2 \alpha$ expression and increased tumorigenicity is also observed in colon cancer cells $^{17}$ and gastric adenocarcinoma ${ }^{20}$.

\section{AP-2 $\beta$}

AP-2 $\beta$ plays a critical role in development of ductus arteriosus and limb patterning ${ }^{21}$. TFAP $2 B$ mutation leads to nonsyndromic patent ductus arteriosus and Char syndrome, which is characterized by patent ductus arteriosus, facial dysmorphism and abnormalities of the fifth finger ${ }^{6,22}$. Besides, AP- $2 \beta$ is also involved in glucose and fat metabolism. Gene variations of TFAP $2 B$ are related to insulin resistance and type 2 diabetes mellitus ${ }^{7}$. It has also been reported to influence adiposity-related conditions and intrauterine growth ${ }^{23}$.

Similar to AP- $2 \alpha$, reduced or increased TFAP2B expression has also been reported in human cancers. Enhanced expression of AP-2 $\beta$ has been reported in lobular carcinoma in situ(LCIS) and invasive lobular breast cancer ${ }^{24}$. In addition, overexpression of AP-2 $\beta$ is related to poor prognosis in lung adenocarcinoma ${ }^{25}$ and papillary thyroid cancer ${ }^{26}$, while decreased expression seems to be correlated with unfavorable prognosis and adverse patient outcome in neuroblastoma ${ }^{15}$ and endometrial carcinoma ${ }^{27}$.

\section{AP- $2 \gamma$}

Studies have suggested the relationship between AP$2 \gamma$ and lung carcinoma. AP- $2 \gamma$ acts as an oncogenic factor promoting lung tumorigenesis ${ }^{28}$ and has a critical role in lung cancer development ${ }^{29}$. Besides, in lung carcinoma cells, the expression of AP- $2 \gamma$ is detected to be increased ${ }^{12}$, which inhibits the expression of GADD45B and PMAIP1, then promotes proliferation and motility of cells in nonsmall cell lung cancer ${ }^{30}$.

Interestingly, AP- $2 \gamma$ has been reported to have opposite effects on breast cancer: it firstly delayed tumor initiation, however then promoted tumor progression ${ }^{13}$. TFAP2C has been found to influence development of the luminal cell type during mammary development and to act as a critical transcriptional regulator, which maintains the luminal phenotype ${ }^{31}$. TFAP2C regulates expression of many genes in breast cancer. TFAP2C is firstly reported to induce high expression of ERBB2(Her2) and ESR1 (ERa), which influences hormone response in breast cancer cells ${ }^{8}$. Furthermore, studies have suggested TFAP2C coordinates the expression of some other primary target genes, including FOXA1, WWOX, GREB1, CDH2, HPSE, IGSF11, etc $^{32,33}$. The expression level of TFAP2C also coordinates with treatment response and survival rate of patients with breast cancer. High expression of TFAP2C has been reported to repress $C D 44$, a basal-associated gene of breast cancer, and lead to a higher rate of pathologic complete response after neoadjuvant chemotherapy ${ }^{34}$. However, another research suggests that overexpression of TFAP2C is associated with a shorter survival beyond 10 years of diagnosis $^{35}$.

\section{AP-2 $\delta$}

Researches on AP- $2 \delta$ and TFAP $2 D$ are relatively rare. Compared with other members of AP-2 family, AP- $2 \delta$ seems to influence mammalian development in a different way. Expression of AP- $2 \delta$ has been reported in retina, the central nervous system, and the developing heart, while 
the neural crest, facial mesenchyme, and limbs hardly shows any expression ${ }^{4}$. Expression of $\mathrm{AP}-2 \delta$ in ganglion cells promotes the fine-tuning of axonal growth in the developing retina ${ }^{36}$. Besides, loss of AP- $2 \delta$ coordinates with reduced axonal projections to the superior colliculus ${ }^{37}$.

AP-2 $\delta$ has also been found in tissue of the prostate ${ }^{38}$. Moreover, in aggressive tumor phenotype of prostate cancer, upregulation of AP- $2 \delta$ is detected ${ }^{39}$.

\section{AP-2E}

Many studies of AP- $2 \varepsilon$ concentrate on its prediction value on patients' response to chemotherapy and outcome in colorectal cancer. However, this issue is still under dispute. Elbert's study indicates that hypermethylation of TFAP2E is correlated with resistance to chemotherapy in colorectal cancer ${ }^{40}$, while another research shows the response to chemotherapy cannot be predicted by the level of methylation ${ }^{41}$. As for the value of predicting prognosis, there's also no consensus. Some studies show hypermethylation is associated with survival advantage $e^{42}$ while the correlation with poorer overall and disease-free survival has also been reported ${ }^{43}$.

In addition, $\mathrm{AP}-2 \varepsilon$ is also associated with human neuroblastoma. It is reported that AP- $2 \varepsilon$ is involved in the regulation of DNA damage response in neuroblastoma cells ${ }^{44}$.

The AP-2 family of transcription factors play irreplaceable roles in embryogenesis, body formation and development. TFAP $2 A$ and TFAP $2 B$ are also involved in regulation of endocrine processes. In addition, the family have also been found to be involved in tumorigenesis, development and prognosis of a variety of human cancers. And now they are still being studied extensively in human cancer. Considering the influence of AP-2 family on tumor type, therapeutic response and prognosis, especially as demonstrated in breast and colorectal cancers, these studies may bring new idea to the diagnosis, classification, treatment and prognosis of cancer. Meanwhile, there are still many controversial issues, including AP-2ع's prediction value on patients' response to chemotherapy and outcome in colorectal cancer, to be studied.

\section{Acknowledgment}

This work was supported by the Science \& Technology Department of Sichuan Province, No.2021YFS0111. The views expressed are those of the authors and not necessarily those of the Science \& Technology Department of Sichuan Province. We apologize for not being able to cite all the publications related to this topic due to space constraints of the journal.

\section{Abbreviations}

TFAP: transcription factor activating proteins.

KRT16: gene of keratin 16, a type of I cytokeratin
GADD45B: gene of growth arrest and DNA-damageinducible beta

PMAIP1: gene of phorbol-12-myristate-13-acetateinduced protein 1

ERBB2: V-Erb-B2 Avian Erythroblastic Leukemia Viral Oncogene Homolog 2

Her2: gene of human epidermal growth factor receptor-2

ESR1: gene of estrogen receptor 1

$E R a:$ gene of estrogen receptor $\alpha$

FOXA1: gene of forkhead box protein A1

WWOX: gene of WW domain-containing oxidoreductase

GREB1: gene of growth regulation by estrogen in breast cancer 1

CDH2: gene of N-cadherin

HPSE: gene of heparanase

IGSF11: gene of immunoglobulin superfamily member 11

CD44: gene of cluster of differentiation 44

\section{References}

1. Eckert D, Buhl S, Weber S, et al. The AP-2 family of transcription factors. Genome Biol. 2005; 6(13): 246.

2. Zhao F, Satoda M, Licht JD, et al. Cloning and characterization of a novel mouse AP-2 transcription factor, AP-2delta, with unique DNA binding and transactivation properties. J Biol Chem. 2001; 276(44): 40755-40760.

3. Zhang J, Hagopian-Donaldson S, Serbedzija G, et al. Neural tube, skeletal and body wall defects in mice lacking transcription factor AP2. Nature. 1996; 381(6579): 238-241.

4. Zhao F, Lufkin T, Gelb BD. Expression of Tfap2d, the gene encoding the transcription factor Ap-2 delta, during mouse embryogenesis. Gene Expr Patterns. 2003; 3(2): 213-217.

5. Milunsky JM, Maher TA, Zhao G, et al. TFAP2A mutations result in branchio-oculo-facial syndrome. Am J Hum Genet. 2008; 82(5): 11711177.

6. Satoda M, Zhao F, Diaz GA, et al. Mutations in TFAP2B cause Char syndrome, a familial form of patent ductus arteriosus. Nat Genet. 2000; 25(1): 42-46.

7. Maeda S, Tsukada S, Kanazawa A, et al. Genetic variations in the gene encoding TFAP2B are associated with type 2 diabetes mellitus. J Hum Genet. 2005; 50(6): 283-292.

8. Woodfield GW, Horan AD, Chen Y, et al. TFAP2C controls hormone response in breast cancer cells through multiple pathways of estrogen signaling. Cancer Res. 2007; 67(18): 8439-8443.

9. Anttila MA, Kellokoski JK, Moisio KI, et al. Expression of transcription factor AP-2alpha predicts survival in epithelial ovarian cancer. Br J Cancer. 2000; 82(12): 1974-1983.

10. Bar-Eli M. Role of AP-2 in tumor growth and metastasis of human melanoma. Cancer Metastasis Rev. 1999; 18(3): 377-385.

11. Heimberger AB, McGary EC, Suki D, et al. Loss of the AP-2alpha transcription factor is associated with the grade of human gliomas. Clin Cancer Res. 2005; 11(1): 267-272. 
12. Cheng C, Ai Z, Zhao L. Comprehensive analysis of the expression and prognosis for TFAP2 in human lung carcinoma. Genes Genomics. 2020; 42(7): 779-789.

13. Jäger R, Friedrichs N, Heim I, et al. Dual role of AP-2gamma in ErbB2-induced mammary tumorigenesis. Breast Cancer Res Treat. 2005; 90(3): 273-280.

14. Shi D, Xie F, Zhang Y, etal. TFAP2A regulates nasopharyngeal carcinoma growth and survival by targeting HIF-1 $\alpha$ signaling pathway. Cancer Prev Res (Phila). 2014; 7(2): 266-277.

15. Ikram F, Ackermann S, Kahlert Y, et al. Transcription factor activating protein 2 beta (TFAP2B) mediates noradrenergic neuronal differentiation in neuroblastoma. Mol Oncol. 2016; 10(2): 344-359.

16. Sun J, Du N, Li J, et al. Transcription Factor AP2ع: A Potential Predictor of Chemoresistance in Patients With Gastric Cancer. Technol Cancer Res Treat. 2016; 15(2): 285-295.

17. Schwartz B, Melnikova VO, Tellez C, et al. Loss of AP-2alpha results in deregulation of E-cadherin and MMP-9 and an increase in tumorigenicity of colon cancer cells in vivo. Oncogene. 2007; 26(28): 4049-4058.

18. Yuanhua L, Pudong Q, Wei Z, et al. TFAP2A Induced KRT16 as an Oncogene in Lung Adenocarcinoma via EMT. Int J Biol Sci. 2019; 15(7): 1419-1428.

19. Douglas DB, Akiyama Y, Carraway H, et al. Hypermethylation of a small CpGuanine-rich region correlates with loss of activator protein2alpha expression during progression of breast cancer. Cancer Res. 2004; 64(5): 1611-1620.

20. Wang W, Lv L, Pan K, et al. Reduced expression of transcription factor AP- $2 \alpha$ is associated with gastric adenocarcinoma prognosis. PloS One. 2011; 6(9): e24897.

21. Zhao F, Bosserhoff AK, Buettner R, et al. A heart-hand syndrome gene: Tfap2b plays a critical role in the development and remodeling of mouse ductus arteriosus and limb patterning. PloS One. 2011; 6(7): e22908.

22. Khetyar M, Syrris P, Tinworth L, et al. Novel TFAP2B mutation in nonsyndromic patent ductus arteriosus. Genet Test. 2008; 12(3): 457-459.

23. Comasco E, Iliadis SI, Larsson A, et al. Adipocytokines levels at delivery, functional variation of TFAP $2 \beta$, and maternal and neonatal anthropometric parameters. Obesity (Silver Spring). 2013; 21(10): 2130-2137.

24. Raap M, Gronewold M, Christgen $\mathrm{H}$, et al. Lobular carcinoma in situ and invasive lobular breast cancer are characterized by enhanced expression of transcription factor AP-2 $\beta$. Lab Invest. 2018; 98(1): 117-129.

25. Fu L, Shi K, Wang J, et al. TFAP2B overexpression contributes to tumor growth and a poor prognosis of human lung adenocarcinoma through modulation of ERK and VEGF/PEDF signaling. Mol Cancer. 2014; 13: 89.

26. A AA, O AH, L MG, et al. TFAP2B, AP-1 and JAZF1 Expression in Tissues of Papillary Thyroid Carcinoma Patients; Clinical, Pathological and Prognostic Values. Asian Pac J Cancer Prev. 2020; 21(8): 2415-2421.

27. Cui P, Shi K, Cui HX, et al. Correlation between transcription factor activator protein-2 $\beta$ (TFAP- $2 \beta$ ) and endometrial carcinoma. Eur J Gynaecol Oncol. 2015; 36(3): 268-273.
28. Kim W, Kim E, Lee S, et al. TFAP2C-mediated upregulation of TGFBR1 promotes lung tumorigenesis and epithelial-mesenchymal transition. Exp Mol Med. 2016; 48(11): e273.

29. Kang J, Kim W, Lee S, et al. TFAP2C promotes lung tumorigenesis and aggressiveness through miR-183- and miR-33a-mediated cell cycle regulation. Oncogene. 2017; 36(11): 1585-1596.

30. Do H, Kim D, Kang J, et al. TFAP2C increases cell proliferation by downregulating GADD45B and PMAIP1 in non-small cell lung cancer cells. Biol Res. 2019; 52(1): 35.

31. Cyr AR, Kulak MV, Park JM, et al. TFAP2C governs the luminal epithelial phenotype in mammary development and carcinogenesis. Oncogene. 2015; 34(4): 436-444.

32. Woodfield GW, Chen Y, Bair TB, et al. Identification of primary gene targets of TFAP2C in hormone responsive breast carcinoma cells. Genes Chromosomes Cancer. 2010; 49(10): 948-962.

33. Ailan $\mathrm{H}$, Xiangwen X, Daolong R, et al. Identification of target genes of transcription factor activator protein 2 gamma in breast cancer cells. BMC Cancer. 2009; 9: 279.

34. Spanheimer PM, Askeland RW, Kulak MV, et al. High TFAP2C/low CD44 expression is associated with an increased rate of pathologic complete response following neoadjuvant chemotherapy in breast cancer. J Surg Res. 2013; 184(1): 519-525.

35. Perkins SM, Bales C, Vladislav T, et al. TFAP2C expression in breast cancer: correlation with overall survival beyond 10 years of initial diagnosis. Breast Cancer Res Treat. 2015;152(3):519-531.

36. Li X, Monckton EA, Godbout R. Ectopic expression of transcription factor AP-2 $\delta$ in developing retina: effect on PSA-NCAM and axon routing. J Neurochem. 2014; 129(1): 72-84.

37. Li X, Gaillard F, Monckton EA, et al. Loss of AP-2delta reduces retinal ganglion cell numbers and axonal projections to the superior colliculus. Mol Brain. 2016; 9(1): 62.

38. Cheng $\mathrm{C}$, Ying $\mathrm{K}, \mathrm{Xu} \mathrm{M}$, et al. Cloning and characterization of a novel human transcription factor AP-2 beta like gene (TFAP2BL1). Int J Biochem Cell Biol. 2002; 34(1): 78-86.

39. Fraune C, Harms L, Büscheck F, et al. Upregulation of the transcription factor TFAP2D is associated with aggressive tumor phenotype in prostate cancer lacking the TMPRSS2:ERG fusion. Mol Med. 2020; 26(1): 24.

40. Ebert MP, Tänzer M, Balluff B, etal. TFAP2E-DKK4 and chemoresistance in colorectal cancer. N Engl J Med. 2012; 366(1): 44-53.

41. Murcia O, Jover R, Egoavil C, et al. TFAP2E Methylation and Expression Status Does Not Predict Response to 5-FU-based Chemotherapy in Colorectal Cancer. Clin Cancer Res. 2018; 24(12): 2820-2827.

42. Zhang ZM, Wang Y, Huang R, et al. TFAP2E hypermethylation was associated with survival advantage in patients with colorectal cancer. J Cancer Res Clin Oncol. 2014; 140(12): 2119-2127.

43. Beggs AD, Dilworth MP, Domingo E, et al. Methylation changes in the TFAP2E promoter region are associated with BRAF mutation and poorer overall \& disease free survival in colorectal cancer. Oncoscience. 2015; 2(5): 508-516.

44. Hoshi R, Watanabe Y, Ishizuka Y, et al. Depletion of TFAP2E attenuates adriamycin-mediated apoptosis in human neuroblastoma cells. Oncol Rep. 2017; 37(4): 2459-2464. 